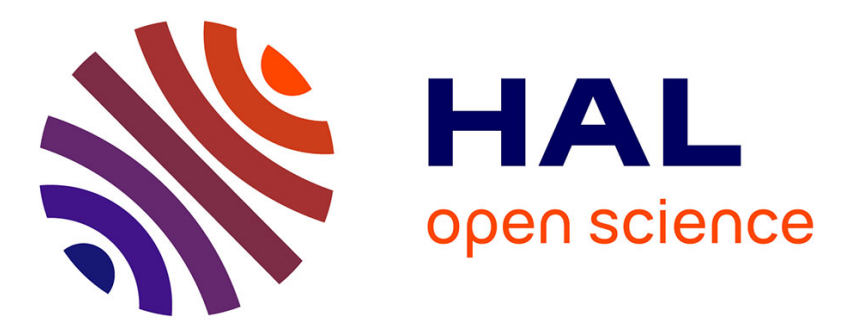

\title{
Speckle pattern creation methods for two-dimensional digital image correlation strain measurements applied to mechanical tensile tests up to $700^{\circ} \mathrm{C}$
}

Linh Phuong Luong, Rébecca Bonnaire, Jean-Noël Périé, Quentin Sirvin, Luc Penazzi

\section{To cite this version:}

Linh Phuong Luong, Rébecca Bonnaire, Jean-Noël Périé, Quentin Sirvin, Luc Penazzi. Speckle pattern creation methods for two-dimensional digital image correlation strain measurements applied to mechanical tensile tests up to $700^{\circ}$ C. Strain, 2021, 57 (5), pp.1-19/e12388. 10.1111/str.12388 . hal03244402

\section{HAL Id: hal-03244402 https://imt-mines-albi.hal.science/hal-03244402}

Submitted on 12 Jul 2021

HAL is a multi-disciplinary open access archive for the deposit and dissemination of scientific research documents, whether they are published or not. The documents may come from teaching and research institutions in France or abroad, or from public or private research centers.
L'archive ouverte pluridisciplinaire HAL, est destinée au dépôt et à la diffusion de documents scientifiques de niveau recherche, publiés ou non, émanant des établissements d'enseignement et de recherche français ou étrangers, des laboratoires publics ou privés. 


\title{
Speckle pattern creation methods for two-dimensional digital image correlation strain measurements applied to mechanical tensile tests up to $700^{\circ} \mathrm{C}$
}

\author{
Phuong Luong ${ }^{1} \odot \quad$ | Rébecca Bonnaire ${ }^{1}$ ( ) Jean-Noel Périé ${ }^{2}$ | | \\ Quentin Sirvin $^{1} \odot$ | Luc Penazzi ${ }^{1} \oplus$
}

${ }^{1}$ Institut Clément Ader (ICA); Université de Toulouse; CNRS, IMT Mines Albi, INSA, ISAE-SUPAERO, UPS; Campus Jarlard, 81013 Albi, France

${ }^{2}$ Institut Clément Ader (ICA); Université de Toulouse; CNRS, UPS, IMT Mines Albi, INSA, ISAE-SUPAERO; 3 rue Caroline Aigle, 31400 Toulouse, France

Correspondence

Phuong Luong, Institut Clément Ader, IMT Mines Albi, Campus Jarlard, 81013 Albi, France.

Email: linh_phuong.luong@mines-albi.fr

\begin{abstract}
The purpose of this study is to develop novel speckle pattern techniques for digital image correlation (DIC) kinematic measurements of mechanical tests at high temperatures, typically from 400 to $700^{\circ} \mathrm{C}$. In this context, the speckle pattern should not only meet morphological criteria (size, density, distance) in order to improve spatial resolution, but it should also present a high contrast and resist high temperature and strain levels. To find a speckle pattern matching these specifications, a comparison was performed on six types of speckle made using different techniques. First, a computer-generated speckle pattern that meets DIC criteria was numerically designed to produce six types of speckle pattern. Next, the speckle patterns produced using these six techniques were compared in terms of speckle morphology, image quality and adherence to titanium alloy TA6V material at high temperatures. From 25 to $600^{\circ} \mathrm{C}$, the speckle pattern made by the technique combining anodisation and laser engraving named M5 technique gave the best contrast (highest value of mean intensity gradient [MIG] and Shannon entropy value) and the adherence of $200 \%$ of strain measurements to the TA6V material. At $700^{\circ} \mathrm{C}$, speckle image quality is considerably reduced due to oxidation of the titanium alloy, and this may not be suitable for DIC measurements. Only the speckles produced by painting in which the paint plays a protective role provide with a better speckle contrast compared with other techniques. However, these speckle patterns enable only a strain measurement of $22 \%$ by the DIC method. This article concludes with guidelines for producing a speckle pattern suitable for hightemperature mechanical tests.
\end{abstract}

\section{KEYWOR D S}

digital image correlation, high temperature, speckle pattern, tensile test, titanium alloy 


\section{INTRODUCTION}

In the aerospace industry, analysis of the shape distortions of complex parts, for example, pylons or air inlets, requires full-field kinematic measurement (displacement and strain) of the object during the cooling phase, from high temperature (typically around $700^{\circ} \mathrm{C}$ here) to room temperature $\left(25^{\circ} \mathrm{C}\right) .{ }^{[1]}$ Thanks to its simple implementation and versatility, digital image correlation (DIC) has been widely employed in experimental mechanics to retrieve full-field kinematic information. ${ }^{[2]}$ It is nowadays considered to be a 'reference' among optically based image processing techniques.

In DIC, the speckle pattern is one of the most important parameters. A 'good' speckle pattern enhances the spatial resolution and reduces measurement errors. ${ }^{[2-6]}$ Studies of speckles by Reu et al. show that a 'good' speckle pattern should meet criteria relating to speckle radius, ${ }^{[3]}$ speckle distance, ${ }^{[4]}$ speckle density ${ }^{[5]}$ and high contrast. ${ }^{[6]}$ Furthermore, it must resist high temperatures and ensure good adherence to specimens throughout mechanical testing.

The search for a 'good' speckle pattern still presents challenges, and many studies are still being conducted into speckle creation at high temperatures. For example, Turner et al., ${ }^{[7]}$ Lyons et al., ${ }^{[8]}$ Pan et al., ${ }^{[9]}$ Hammer et al. ${ }^{[10]}$ and Chartrel et al. ${ }^{[11]}$ used high-temperature paint to create speckles. Speckles made by airbrush or spraying techniques have various sizes, from $10 \mu \mathrm{m}$ to $1 \mathrm{~mm}$, with an image resolution of $20-50 \mu \mathrm{m} /$ pixel. $^{[12]}$ The speckle radius produced by painting techniques depends on users and tools (nozzle tips and pressure). Speckle production is uncontrolled and not repeatable. Furthermore, these speckles become detached from the material surface when subjected to high strain, thus disrupting the correlation process.

To overcome the speckle spallation problem, three new methods have been developed in recent years. First, Vautrot et al. ${ }^{[13]}$ and Sirvin et al. ${ }^{[14]}$ developed electrochemical marking on metallic samples allowing high-strain measurements. The speckle pattern consists of permanent dots made from a thin oxide layer of a different colour to the metal surface. The technique can withstand high plastic strains (until the material reaches its necking state), suggesting a new perspective. However, the speckle radius is large: more than 20 pixels, equivalent to $500 \mu \mathrm{m}$. The laser engraving technique provides an alternative solution. Hu et al. ${ }^{[15]}$ used a laser beam to create a speckle pattern on metallic surfaces. Its radius, approximately $5 \mu \mathrm{m}$, is better in terms of spatial resolution. However, possible modifications to the microstructure and/or mechanical behaviour of the materials were not investigated. The third method is the laser speckle, made by scattered light and a rough material surface. For example, Zheng et al. ${ }^{[16]}$ used the laser speckle method to measure the plastic strain of thin $(0.5 \mathrm{~mm})$ aluminium sheet at $400^{\circ} \mathrm{C}$. This study showed a good agreement between strains measured by the laser speckle and those measured by spraying. Nevertheless, the laser speckle can only be created on a rough surface. Furthermore, it can only be used for the two-dimensional digital image correlation (2D-DIC). It is indeed not possible to use laser speckle for stereo-DIC because images captured from two points of view are by nature totally uncorrelated. Finally, due to the modification of laser speckle during loading, the reference image must be regularly updated, and it is likely difficult to measure large strains.

Table 1 summarises different studies of speckle techniques for high-temperature mechanical tests. These new speckle methods have some limitations: Speckles are too big or not repeatable or require a rough sample surface. This study aims to develop controllable and repeatable patterns that meet the criteria of a 'good' speckle and resist high strain at high temperature. This article is organised as follows: First, Section 2 details the speckle generator, mask production, speckle production techniques and criteria for evaluating speckle quality. Next, Section 3 presents possible methods for making repeatable speckles from a numerical speckle pattern followed by an assessment of speckle quality at three temperatures $\left(25,600\right.$ and $\left.700^{\circ} \mathrm{C}\right)$. Based on these results, speckles giving the best contrast are chosen for their adherence on the specimen in high-temperature tensile tests. Section 4 suggests an appropriate speckle technique that balances 'good' speckle criteria and material adherence as a guideline for choosing the best speckle for mechanical tests at high temperatures. Section 5 concludes and suggests perspectives for further studies.

\section{2 | MATERIALS AND METHODS}

\section{1 | Materials}

The current study involves creating speckles on Ti-6Al-4V (TA6V) alloy samples. The microstructure of this alloy at room temperature contains two phases: the $\beta$-phase is rich in vanadium, while the $\alpha$-phase contains $4 \%$ of aluminium. No phase transformation occurs in TA6V between 400 and $700^{\circ} \mathrm{C}$. At room temperature, Young's modulus of TA6V is $110 \pm 20 \mathrm{GPa}^{[17]}$ It should be noted that TA6V is easily oxidised. The colour of the TA6V oxide layer changes with 
TA B LE 1 Summary of speckle preparation methods used for mechanical tests within the range of hot stamping temperatures

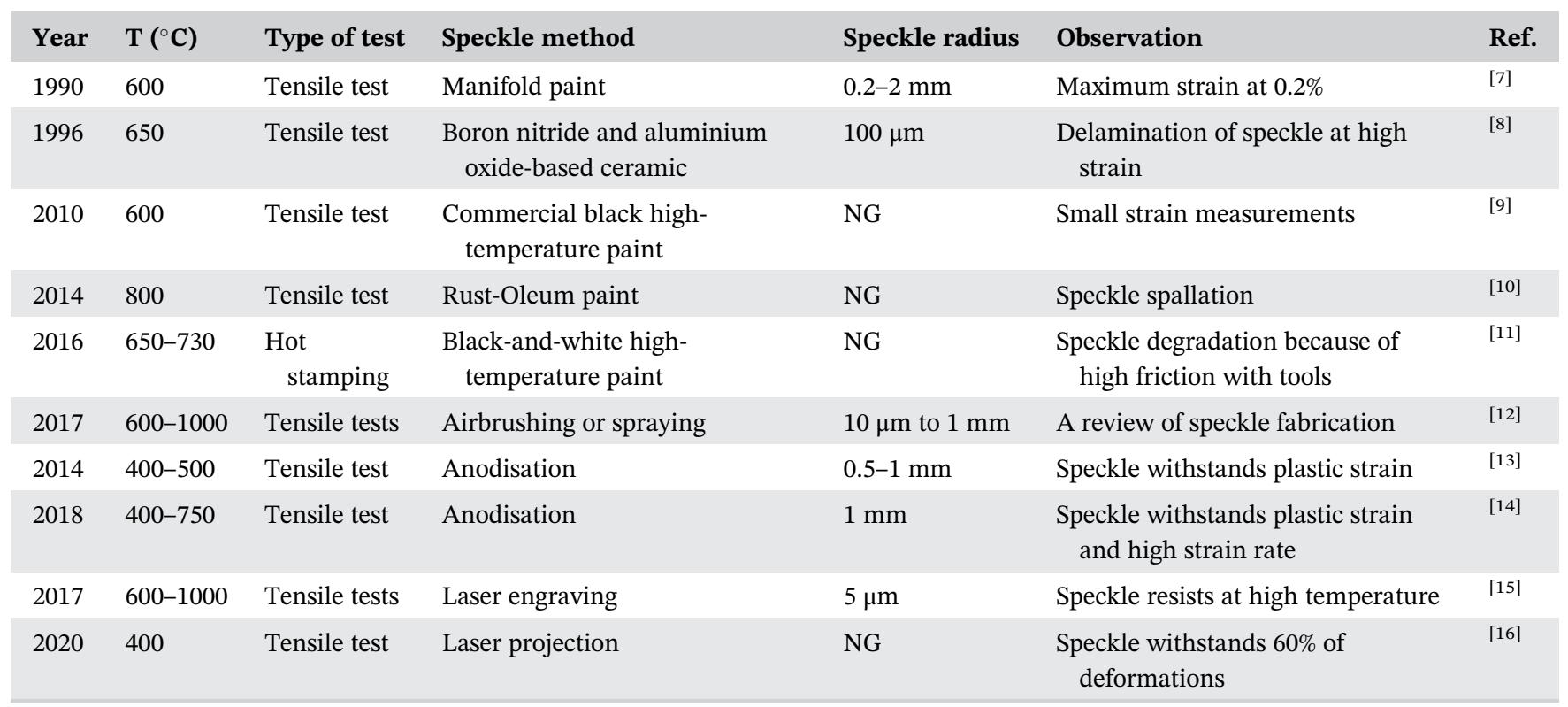

Abbreviation: NG, not given.
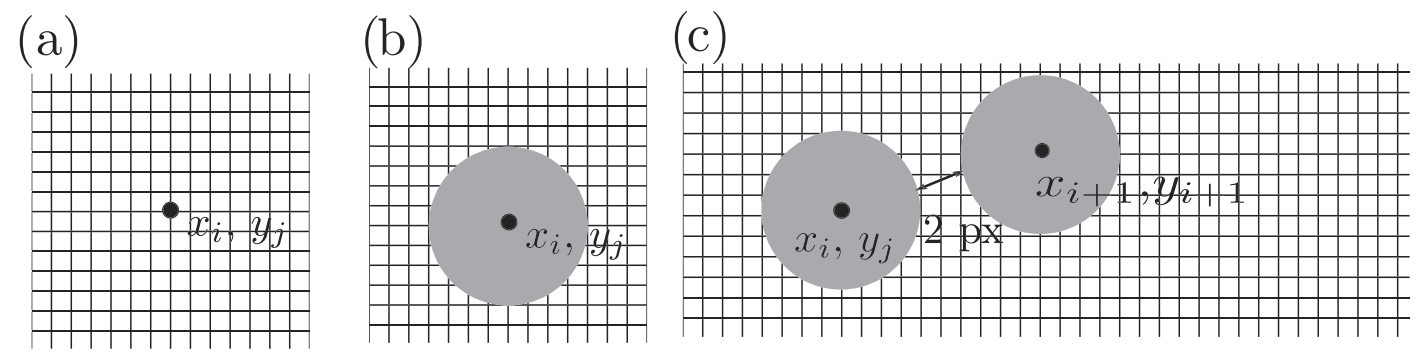

F I G URE 1 Steps to generate a numerical speckle. (a) A random lattice point $n k$ is generated with coordinates $n<k>(x, y<j>)$. (b) The spot is created by assigning the grey level $=1$ within the ZOI. (c) The program goes on to generate the second spot at a distance of two pixels from the first one

thickness, due to interference between the oxide layer and light wavelengths. This characteristic is useful when creating a speckle pattern using an anodisation process.

\section{2 | Numerical creation of a repeatable speckle}

A computer-generated speckle image (binary image) was developed to make a repeatable speckle. The numerical pattern creation process was inspired by the work of Orteu et al. ${ }^{[18]}$ The speckle pattern was circular dots with a radius of seven pixels (equivalent to $105 \mu \mathrm{m}$ ), distance was two pixels $(30 \mu \mathrm{m})$, and density was 0.4 . The speckle was generated using the Matlab software (MathWorks Inc., Massachusetts, USA).

The algorithm for the numerical speckle is presented as follows (Figure 1):

- Initiation: The matrix size $(m \times n)$, speckle radius $r$, distance between dots $d$ and speckle density $\rho$ were defined. The area of one dot was calculated using Equation 1:

$$
A=\pi \cdot r^{2}
$$

and it was assigned a greyscale level of 1. 
- Looping: A random lattice point $n_{k}\left(x_{i}, y_{j}\right)$, where $i=1: n$ and $j=1: m$, was generated on the matrix (Figure 1a). The first speckle dot with area $A$ was created by setting the greyscale level equal to 1 (Figure $1 \mathrm{~b}$ ). The next dots were created if they were at least two pixels away from any others (Figure 1c). This process was stopped when the speckle density (the ratio of greyscale level 1 pixels to total pixels in the matrix) was around 0.4 ( $40 \%$ coverage).

Figure 2 shows the speckle criteria of the numerical speckle: speckle radius: seven pixels $(105 \mu \mathrm{m})$, speckle distance: two pixels $(30 \mu \mathrm{m})$ and speckle density: 0.4.

\section{3 | Speckle preparation methods}

Six types of speckle were created from the numerical speckle using six techniques. Speckles were created on TA6V samples. They were classified into two groups ${ }^{[12]}$ : The first included 'additive' techniques using a polymer mask, while the second included 'subtractive' techniques using a laser source. The six techniques were:

- 'Additive' techniques

- Painting (M1)

- Direct anodisation (M2)

- Inverse anodisation (M3)

- 'Subtractive' techniques

- Laser engraving (M4)

- Combined laser engraving and anodisation (M5)

- Combined laser engraving and painting (M6)

The first three techniques (M1, M2, M3) require the creation of a polymer mask, which enables replication of the numerical speckle. The pattern (holes) on the polymer mask, which should have a similar size to its numerical counterpart, were drilled on a thin polymer film by an additive layer manufacturing machine using the laser source (an IPG Photonics fibre with a beam radius of between 70 and $100 \mu \mathrm{m}$ ) from the SLM 125HL machine (SLM Solutions, Lübeck, Germany). Once the masks had been made, the polymer mask was used to create speckles by matter transfer onto TA6V samples. ${ }^{[19,20]}$ For the last three techniques (M4, M5, M6), speckles were directly engraved onto TA6V samples using the same laser source.

\subsection{1 | Polymer mask production using a laser source}

The thin polymer film was a 0.2-mm-thick vinyl (Vinyl Adhesive Cameo, Silhouette, USA) with a melting point of between 100 and $200^{\circ} \mathrm{C}$. This polymer is in the form of an adhesive paper sheet that can stick onto TA6V samples. The pattern was created by the selective laser melting (SLM) laser source. The variable parameters of the machine were power $P=[10-200 \mathrm{~W}]$, scanning speed $V=[10-200 \mathrm{~mm} / \mathrm{s}]$ and laser trajectory strategy S: (i) hatching (H), (ii) contouring $(\mathrm{C})$ and (iii) a combination of hatching and contouring $(\mathrm{H}+\mathrm{C})$.

Since the laser system has a wide range of values for each parameter (power, scanning speed, laser scan strategy) and the polymer mask has a low melting temperature, the laser's parameters were investigated in order to adapt it to this unconventional material. The results of preliminary experimental tests showed that holes were only created in the polymer if a speckle density of 0.2 and a speckle distance of $320 \mu \mathrm{m}$ were respected. This means that the speckle density

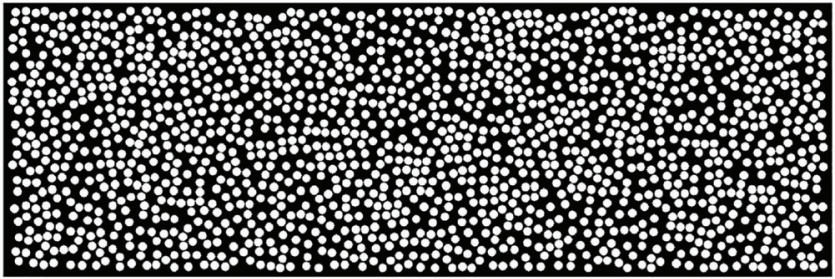

$5 \mathrm{~mm}$
F I G U RE 2 A numerical speckle pattern with respect to speckle criteria: speckle radius $R<\mathrm{c}\rangle=7$ pixels $(\mathrm{R}\langle\mathrm{c}\rangle=105 \mu \mathrm{m})$, speckle distance: $d=2$ pixels $(d=30 \mu \mathrm{m})$ and speckle density $\rho=0.4$ 
obtained using the three techniques M1, M2 and M3 would be lower than that for the numerical speckles. To find a set of laser parameters $(\mathrm{P}, \mathrm{V}, \mathrm{S})$ that would allow the desired pattern to be created on the polymer, nine experiments were conducted, combining different values of the three laser parameters $(\mathrm{P}, \mathrm{V}, \mathrm{S})$. These nine experiments were set up by following the Taguchi orthogonal experimental design. ${ }^{[21]}$ The output of the experiment was the radius $r$ of the hole $(\mu \mathrm{m})$ in the polymer, measured by image processing using Matlab software. Each experiment was conducted three times to check its repeatability. Table 2 presents the laser parameters set up using the Taguchi experimental design and the results. An analysis of the variance in the results from this experimental design was performed to determine the influence of the different laser factors on hole radius.

\subsection{2 | 'Additive' speckle preparation methods: Painting (M1), direct anodisation (M2) and inverse anodisation (M3)}

Polymer masks were used to create speckles using three techniques: painting (M1), direct anodisation (M2) and inverse anodisation (M3). Speckles were created on rectangular TA6V samples (dimensions $=20 \times 8 \mathrm{~mm}$, equivalent to the zone of interest [ZOI] of TA6V specimens used for tensile tests). The technical process for making speckles was based on classical lithography. ${ }^{[12,19,20]}$

For the M1 technique, Vitcas high-temperature silicone resin white paint (Vitcas Ltd., Bristol, UK) was used for the background, while high-temperature $n$-cycloalkane Rust-Oleum High $750^{\circ} \mathrm{C}$ black paint (Rust-Oleum, USA) was used to create the speckle dots. The M3 technique used acrylic varnish (Jelt, RS Components, Beauvais, France) with $<10 \%$ of naphtha heavy petrol to create speckle dots. In the anodisation process, a $1 \mathrm{~g} / \mathrm{L}$ sulphuric acid solution was prepared as the electrolyte. The voltage was generated by a tension generator.

The processes used for the M1, M2 and M3 speckle production techniques were as follows:

- Painting technique (M1): The TA6V sample was first covered in Vitcas paint to create a white background. The polymer mask was then stuck directly onto the sample. The Rust-Oleum black paint was applied to the sample surface using an airbrush (Aztek airbrush model 7778, pressure $=3$ bars, nozzle tip $=0.3 \mathrm{~mm}$ ) to create black dots through holes in the polymer mask. After spraying, the mask was carefully removed.

- Direct anodisation technique (M2): First, the polymer mask was stuck directly onto the rectangular TA6V sample. The metallic sample was then connected to the positive electrode for an electrochemical anodisation process under a tension of $30 \mathrm{~V}$ for $30 \mathrm{~s}$. Black dots were created by oxidation of the TA6V sample, producing a black-on-white speckle. After anodisation, the mask was removed.

TA B LE 2 Nine experimental runs using the Taguchi orthogonal experimental design with three levels and three factors and results to find a set of laser parameters that allow holes to be created in a polymer film

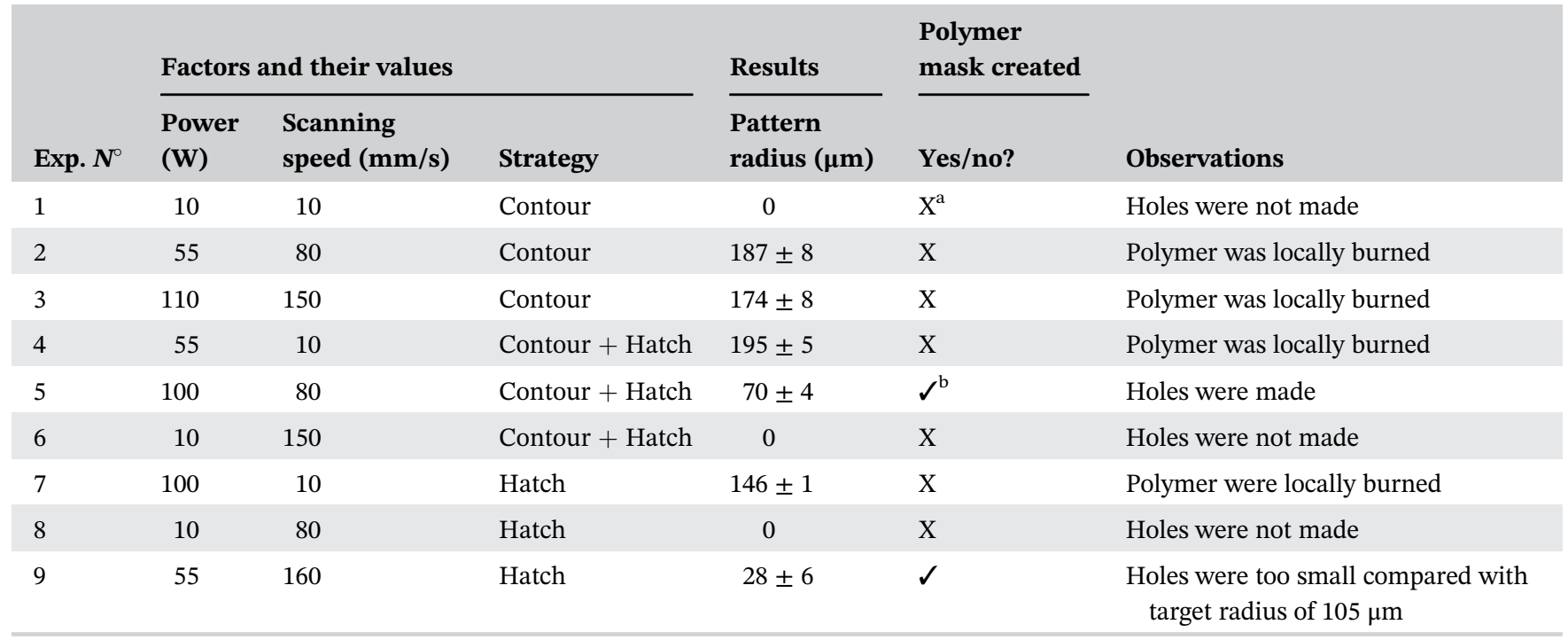

${ }^{\mathrm{a}}$ Not successful.

${ }^{\mathrm{b}}$ Successul. 
- Inverse anodisation technique (M3): First, the polymer mask was stuck directly onto the sample surface. The sample was then covered with the Jelt varnish using the airbrush. The sample was then soaked in the electrolyte bath to allow the anodisation (tension: $20 \mathrm{~V}$, time: $30 \mathrm{~s}$ ) to create a white-on-black speckle pattern. Finally, the Jelt layer was removed using an acetone solvent.

\subsection{3 | 'Subtractive' speckle preparation methods: Laser engraving (M4), combined anodisation} and laser engraving (M5) and combined laser engraving and painting (M6)

Three types of speckle, M4, M5 and M6, were created directly on the surface of TA6V samples $(20 \times 8 \mathrm{~mm})$ by the SLM machine. The laser parameters chosen for our experiment were fixed at power $P: 20 \mathrm{~W}$, scanning speed $V: 150 \mathrm{~mm} / \mathrm{s}$ and strategy S: Hatching + Contouring $(\mathrm{H}+\mathrm{C})$.

Furthermore, to improve the image contrast and to avoid specular reflection from the smooth surface, in technique M5, prior to laser engraving, the TA6V surface was covered by a matt turquoise colour (using the anodisation process $[$ tension $=45 \mathrm{~V}$, time $=30 \mathrm{~s}]$ ). In technique M6, Vitcas white paint was used to avoid reflection and protect the metallic surface from oxidation.

Two tests were performed to ensure that the laser engraving did not deteriorate the microstructure and the mechanical properties of the TA6V samples. Firstly, the surface of the TA6V sample, speckled using the M5 technique, was observed using an optical microscope (Leica GmbH, Germany) and then by scanning electron microscopy (SEM) (FEI, Oregon, USA). Microstructures were observed on a cross-sectional view before and after laser engraving using an SEM with the backscattered electron (BSE) detector to assess microstructural modification in the speckle zone (near the surface). Secondly, two TA6V tensile tests were performed, with and without laser engraving of speckles, to evaluate the mechanical properties of the material before and after laser treatment.

\section{4 | Evaluation of speckle quality at different temperatures}

Speckles made by the six different techniques were evaluated at three temperatures: (i) 25 , (ii) 600 and (iii) $700^{\circ} \mathrm{C}$. Speckle images were recorded by a fronto-parallel camera system. A camera with a 2452-pixel-by-2052-pixel CCD sensor (Pike 505F/B, Allied Vision Technologies, Germany) and an objective with a focal length from 28 to $300 \mathrm{~mm}$ (Sigma, Kanagawa, Japan) were used to capture the images. The focal length and the stand-off distance were set at 300 and $1.2 \mathrm{~m}$, respectively. The magnification was $13.9 \mu \mathrm{m} /$ pixel. The field of view was $34 \times 28.5 \mathrm{~mm}$. The camera aperture was set at $\mathrm{f} / 8$, and exposure time varied from 4 to $19 \mathrm{~ms}$ depending on the speckle-creation technique. A diffused angle white light powered at $300 \mathrm{~W}$ (Dedolight, Dedo Weigert Film GmbH, Germany) was used to illuminate the samples. It should be noted that the light was set up behind the camera and far enough away to avoid having a temperature effect on images (heating of the camera or heatwaves from a light source). Furthermore, it is highlighted that polarisers were not used to reduce the problem of pixel saturations in this study due to material constraints and the narrow observation window ( $15 \mathrm{~mm}$ wide) (Figure 3). Images were captured instantaneously by Vic-Snap

(a)

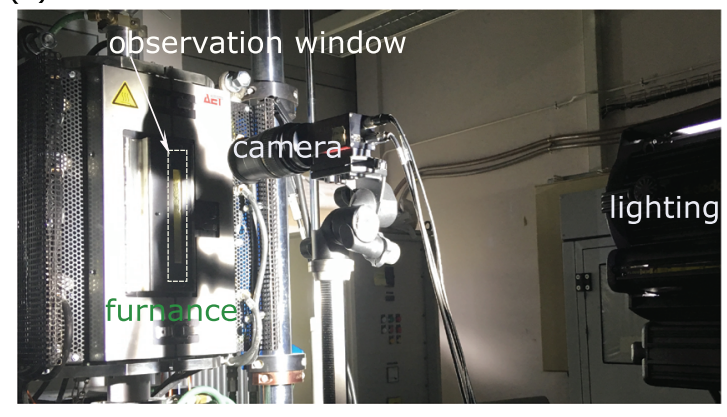

(b)

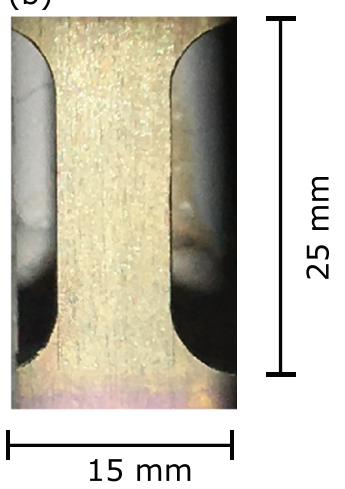

F I G U R E 3 Fronto-parallel camera set-up for measuring speckle images 
software (Correlated Solutions, USA). Images obtained by this method were used to calculate different speckle parameters.

Two categories of parameter were chosen to evaluate speckle quality:

- Morphology via the speckle radius $r$, speckle density $\rho$ and speckle distance $d$

- Image quality, defined by (i) mean intensity gradient (MIG), calculated by Equation $2^{[22]}$; (ii) Shannon entropy, ${ }^{[12]}$ calculated by Equation 3; and (iii) the percentage of saturated pixels

The speckle morphology parameters (radius $r$, density $\rho$, distance $d$ ) were calculated using built-in Matlab image processing functions. The speckle density is the ratio between the total area of speckle dots and the total area of the ZOI. MIG and Shannon entropy were calculated using Equations 2 and 3, respectively.

$$
M I G=\frac{\sum_{i=1}^{m} \sum_{j=1}^{n} \sqrt{f_{x}\left(x_{i}, y_{j}\right)^{2}+f_{y}\left(x_{i}, y_{j}\right)^{2}}}{m \times n}
$$

where $f_{x}\left(x_{i}, y_{j}\right), f_{y}\left(x_{i}, y_{j}\right)$ are the grey-level intensity gradients of pixel $\left(x_{i}, y_{j}\right)$ in the $x$ and $y$ directions; $\mathrm{m}$ (pixels) $\times \mathrm{n}$ (pixels) is the area of the ZOI.

$$
E=\sum_{i=0}^{255} p_{i} \log _{2} p_{i}
$$

where $p_{i}$ is the number of pixels with grey level $i$ in the ZOI.

It should be noted that the entropy might not be the best criterion for the speckle quality assessment. Very small patterns with lots of contrast or added noise will indeed increase the entropy values. In this case, entropy would rank a noisy image better than a low noise image However, in our study, this criterion can be used as a speckle quality indicator, because the pattern is adequately sized (speckle radius is more than three pixels).

The percentage of saturated pixels was defined as the ratio between the number of pixels with a grey level of 255 and the total number of pixels in the ZOI.

The relative reduction in MIG and entropy values at 600 and $700^{\circ} \mathrm{C}$ compared with those at $25^{\circ} \mathrm{C}$ was calculated to evaluate the influence of temperature on speckle image.

\section{5 | Evaluation of speckle adherence at high temperatures}

After evaluating the speckle quality produced by different techniques, four speckles produced by the techniques yielding the best contrast and respecting speckle morphology, that is, M1, M4, M5 and M6, were chosen for the assessment of speckle adherence. The speckle adherence was evaluated by uniaxial tensile tests at $600^{\circ} \mathrm{C}$.

In this experiment, planar specimens measuring $125 \times 25 \times 1.6 \mathrm{~mm}$ were chosen for uniaxial tensile tests. Specimens were heated to $600^{\circ} \mathrm{C}$ by a tensile testing furnace (AET Technologies, France) with a heating rate of $10^{\circ} \mathrm{C} / \mathrm{min}$. During the test, a difference of $2^{\circ} \mathrm{C}$ was observed in the ZOIs of the specimens. The testing machine used is an MTS model 125 (MTS, Minneapolis, USA) with a load capacity of $50 \mathrm{kN}$. The strain rate of mechanical tests was set at $0.001 \mathrm{~s}^{-1}$. Figure 3 presents the experiment set-up to evaluate the adherence of speckle patterns on the material at high temperatures.

Speckle images were captured by the same camera system used for the evaluation of speckle quality at different temperatures. The acquisition frequency was $5 \mathrm{~Hz}$. In order to optimise the dynamic range of greyscale levels, the exposure time was set up to vary from 4 to $16 \mathrm{~ms}$. The longitudinal strain of each specimen was then calculated using the VIC-2D software (Version 6, Correlated Solutions, USA). The limit of adherence of the speckles to the surface sample was determined as the maximum longitudinal strain measured before the first flake of speckles. Table 3 presents the 2D-DIC set-up parameters to measure the strain of the TA6V specimen. These parameters are presented by following the practice guide for the DIC method suggested by the International Digital Image Correlation Society (iDICs). ${ }^{[23]}$ 
T A B LE 3 DIC set-up and VIC-2D set-up parameters for strain measurements in tensile tests

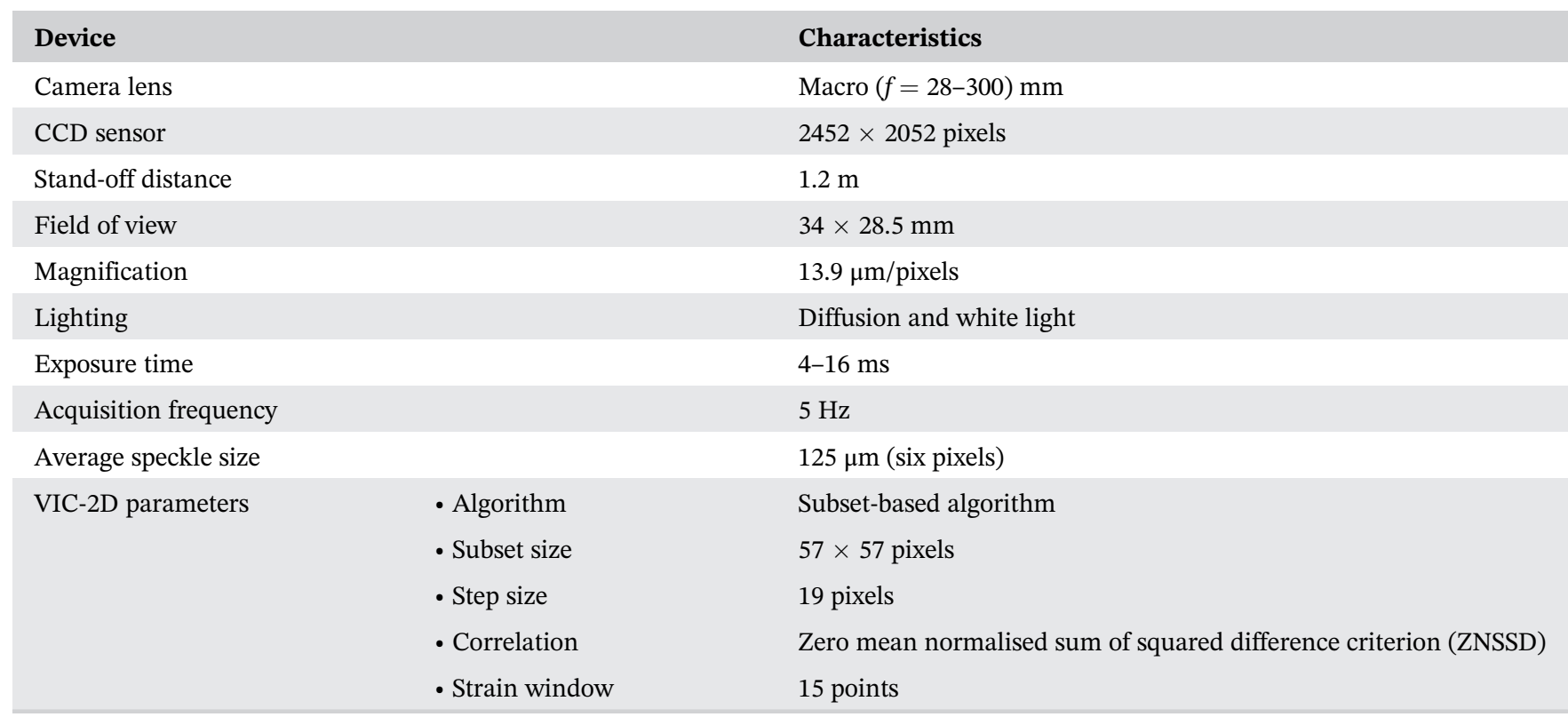

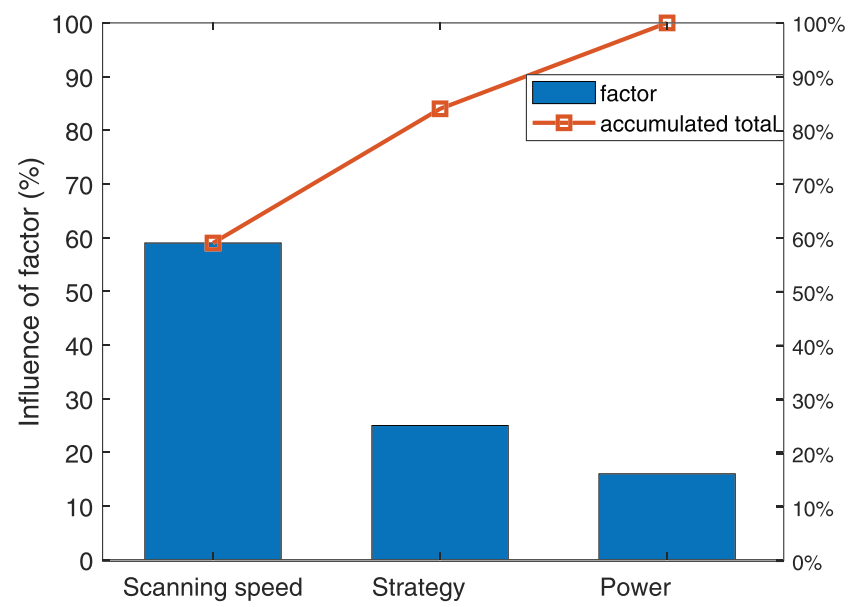

F I G U RE 4 Pareto diagram showing the influence of laser factors on variation in hole radius in the polymer mask

\section{3 | RESULTS}

\section{1 | Creating repeatable speckles at room temperature using six different techniques}

\subsection{1 | Polymer mask production}

The objective of making a polymer mask is to integrate numerical speckle characteristics (size, density and distance) into physical speckle patterns. However, preliminary results on the possibility of creating holes in a polymer sheet show that the speckle density on the polymer had to be reduced to 0.2 and that the speckle distance had to be more than $230 \mu \mathrm{m}$ because of the high level of energy produced by the laser and the relatively low melting temperature of the polyvinyl polymer.

Table 2 presents the mean hole radius values obtained from the nine experiments with different laser parameters. Only two sets of parameters successfully created holes in the polymer. By considering the differences between hole density, hole radius and the numerical pattern, it is likely that the set of laser parameters that would be the best for creating the polymer mask is laser power $P=100 \mathrm{~W}$, scanning speed $V=80 \mathrm{~mm} / \mathrm{s}$ and strategy $=$ contour and hatch. The hole radius obtained with these laser parameters is approximately $70 \mu \mathrm{m}$, which is closest to the numerical size and easiest for paint transfer. Other laser parameters in the experimental design failed to create suitable holes. Indeed, in 
Experiments 1 and 8, no holes were created because of low laser power $(P=10 \mathrm{~W})$. In Experiments 2-4 and 7, holes were made, but the polymer was locally damaged (near the hole borders). In Experiment 9, hole size was about $28 \mu \mathrm{m}$, which is much smaller than the target size.

The analysis of variance shows that all three factors (power, scanning speed and strategy) have a statistically significant influence on polymer hole radius. Figure 4 shows that scanning speed has the greatest effect (60\%), followed by laser strategy, while laser power has the smallest influence on variation in hole radius.

With the results obtained from the experimental design (Table 2), the set of laser parameters $-P=100 \mathrm{~W}$, $V=80 \mathrm{~mm} / \mathrm{s}$ and strategy $=$ contour and hatch-was used to create a polymer mask. However, even with the laser set to $P=55 \mathrm{~W}, V=80 \mathrm{~mm} / \mathrm{s}$, the polymer mask showed considerable local damage that would have been caused by the high energy density of the laser being deposited on a thin polymer (Figure 5a). To create the polymer mask, the laser power was reduced to $30 \mathrm{~W}$. The final laser set up was laser power $=30 \mathrm{~W}$, scanning speed $=80 \mathrm{~mm} / \mathrm{s}$, strategy $=$ contour and hatch. Figure $5 \mathrm{~b}$ shows the polymer mask with dot radius $r=150 \mu \mathrm{m}$, distance $d=320 \mu \mathrm{m}$ and density $=0.2$.

(a)

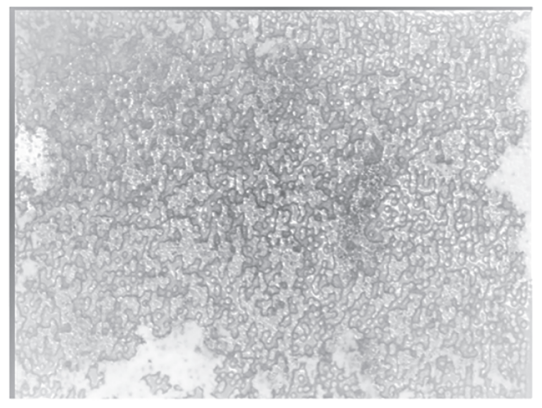

(b)

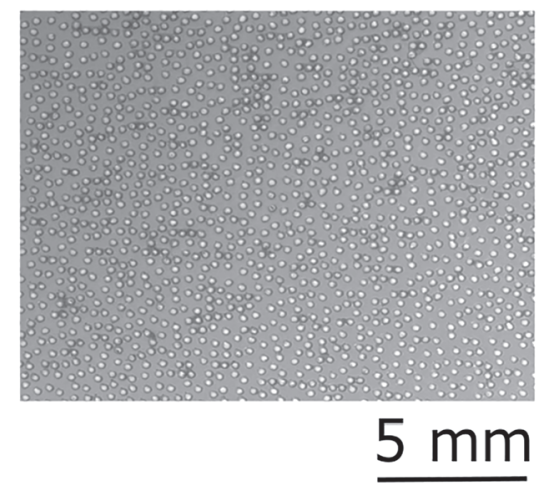

FI G URE 5 (a) Polymer mask obtained with laser parameters: $P=55 \mathrm{~W}, V=80 \mathrm{~mm} / \mathrm{s}$. The polymer is locally burned because of the high energy produced by the laser source. (b) Polymer mask obtained with laser parameters: $P=30 \mathrm{~W}, V=80 \mathrm{~mm} / \mathrm{s}$. Holes are created cleanly in the polymer
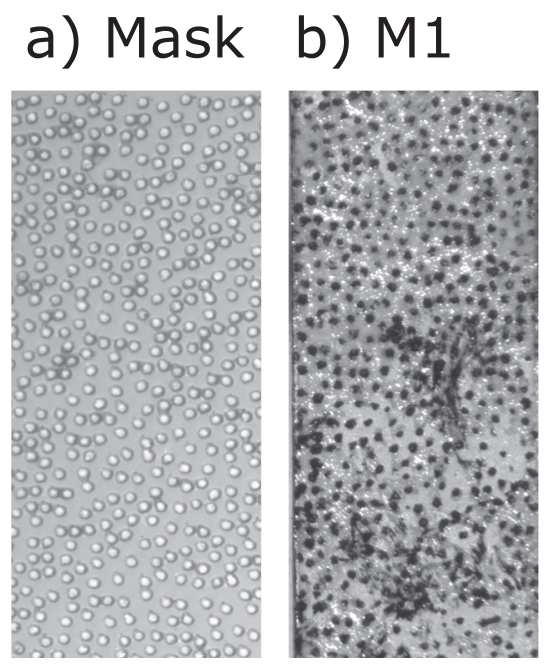
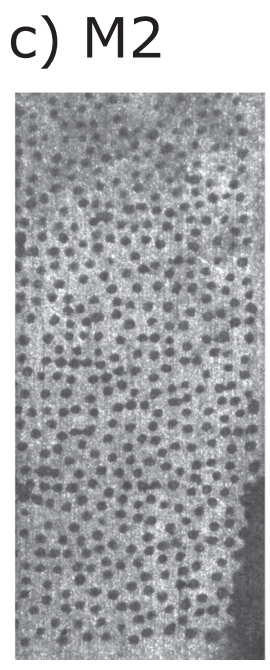
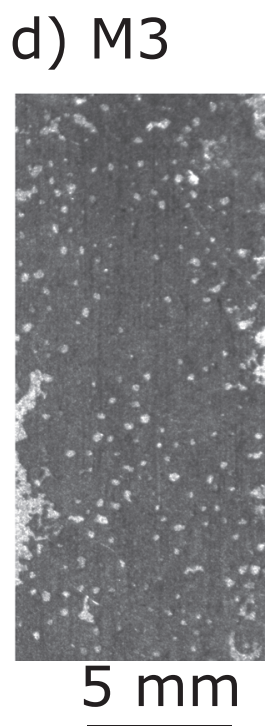

FI G URE 6 (a) Patterns created on the polymer mask used for the 'additive' speckle production techniques. From (b) to (d), speckle images made by (b) M1 (painting), (c) M2 (direct anodisation), (d) M3 (inverse anodisation) at room temperature 


\subsubsection{I 'Additive' speckle preparation methods: Painting (M1), direct anodisation (M2) and inverse anodisation (M3) using a polymer mask}

Figure 6b-d presents three types of speckles and Table 4 compares the radius $r$, distance $d$ and density $\rho$ of speckles produced by techniques M1, M2 and M3.

First, these results show that speckle patterns produced using techniques M1 and M2 most closely replicate the polymer mask pattern. On the other hand, with technique M3, only a few speckle dots are copied onto the TA6V sample. The speckle radius, speckle density and distance between dots made by the three techniques are therefore different. For the first two techniques, speckle radii are similar, $R(\mathrm{M} 1)=151 \pm 230 \mu \mathrm{m}$ and $R(\mathrm{M} 2)=152 \pm 237 \mu \mathrm{m}$, and larger than the target radius $\left(R_{c}=105 \mu \mathrm{m}\right)$, while the radius of speckle dots made by technique M3 is $101 \pm 114 \mu \mathrm{m}$. The speckle densities produced using techniques M1 and M2 are slightly lower than the target density $(\rho=0.24$ and 0.34$)$, while the density is only 0.1 for speckles made using M3. The low speckle density produced using technique M3 is due to the high viscosity of the Jelt varnish that renders matter transfer difficult. The speckle distances produced using the three techniques M1, M2 and M3 are much greater than the numerical distance $d(\mathrm{M} 1)=308 \pm 89 \mu \mathrm{m}, d(\mathrm{M} 2)=294$ $\pm 81 \mu \mathrm{m}, d(\mathrm{M} 3)=296 \pm 89 \mu \mathrm{m}$ but with numerically produced speckles $d=30 \mu \mathrm{m}$. The great distance between speckle dots is explained by the polymer mask pattern distance $d$ (mask) $=320 \mu \mathrm{m}$. After comparing the morphological results for speckles made using the three additive techniques with numerically produced speckles, speckles using technique M2 are the closest to the target parameters.

In addition to the morphological parameters, TA6V surfaces were examined after speckle preparation. It was observed that the 'additive' techniques using the matter transfer process presented a problem with respect to the tape holding down the polymer mask. With technique M1, the TA6V sample presented stains from the adhesive layer of the polymer mask. With techniques M2 and M3, the borders of the TA6V samples were not covered by speckle dots because the polymer mask was not sufficiently well taped onto the sample at the edges. The acid solution was able to penetrate under these edges and oxidised the area.

T A B LE 4 Comparison of speckle radii, speckle distances and speckle densities of six different techniques (M1-M6) with numerical speckles and the polymer mask pattern

\begin{tabular}{|lccc}
\hline Speckle patterns & Speckle radius $\boldsymbol{r}(\boldsymbol{\mu m})$ & Speckle distance $\boldsymbol{d}(\boldsymbol{\mu m})$ & Speckle density $\boldsymbol{\rho}$ \\
\hline Numerical speckles & 105 & 40 & 0.4 \\
\hline Polymer mask pattern & $150 \pm 85$ & 320 & 0.2 \\
\hline M1: painting & $151 \pm 230$ & $308 \pm 89$ & 0.24 \\
\hline M2: direct anodisation & $152 \pm 237$ & $294 \pm 81$ & 0.34 \\
\hline M3: inverse anodisation & $101 \pm 114$ & $296 \pm 89$ & 0.1 \\
\hline M4: laser engraving & $114 \pm 200$ & $185 \pm 70$ & 0.22 \\
\hline M5: combined anodisation and laser & $139 \pm 50$ & $185 \pm 70$ & 0.39 \\
\hline M6: combined paint and laser & $89 \pm 115$ & $185 \pm 70$ & 0.29 \\
\hline
\end{tabular}

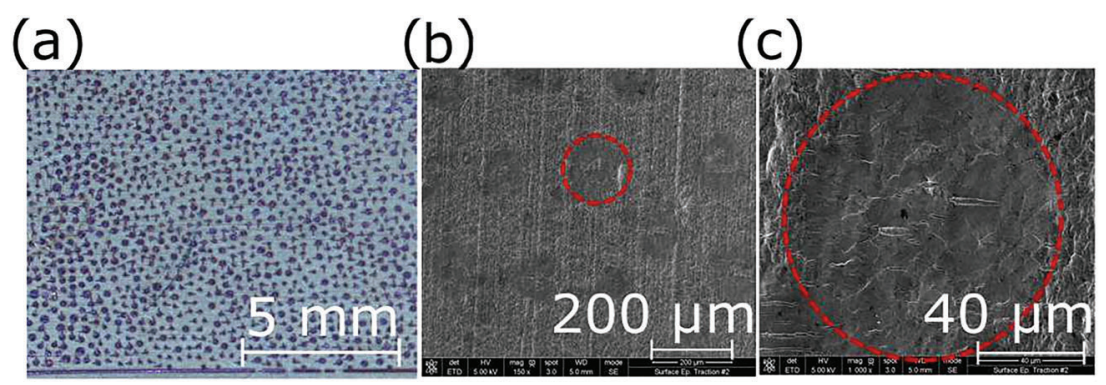

F I G U R E 7 TA6V sample surfaces with speckles prepared using M5 technique (combined anodisation and laser engraving).

(a) Observed using optical microscopy. (b) Observed using SEM. (c) Detail of a speckle dot (in red circle): It has a crater-like shape due to the interaction between the laser and the material 
To summarise, the speckle patterns made using techniques M1 and M2 replicated the pattern of the polymer mask. The density and radii of these speckles were close to the numerical version, but the speckle distance was much greater because of the limitations imposed by the polymer mask. Meanwhile, speckles made by technique M3 presented a small speckle radius and lower density compared with the numerical speckle pattern. Furthermore, the additive techniques presented problems related to the adhesive layer of the polymer mask.

\subsection{3 | 'Subtractive' speckle preparation methods: Laser engraving (M4), combined anodisation and laser engraving (M5) and combined anodisation and painting (M6)}

For the 'subtractive methods', in order to evaluate the interaction between the laser and the material surface, the TA6V surface topography and microstructure were first characterised, and its mechanical behaviour was measured after speckling. Observations using optical microscopy (Figure 7a) and SEM (Figure 7b,c) on a speckled sample after laser treatment show that the speckle dots have crater-like shapes. Some microcracks are also observed on the speckle dots due to rapid cooling during the SLM process (Figure 7c). The microstructure of the cross section of material before (Figure 8a) and after patterning (Figure 8b,c) were compared. SEM observations of the cross section of a speckled region (Figure $7 \mathrm{~b}$ ) show that each speckle dot was a molten pool created by laser-metal interaction. The molten pools were approximately $20 \mu \mathrm{m}$ deep (Figure $8 \mathrm{~b}$ ). Due to the rapid cooling in the SLM process, the microstructure of the molten pool (Figure 8c) has changed from its initial equiaxial state (Figure 8a) to needle-like grains (Figure 8c). Furthermore, the mechanical behaviour of TA6V material after laser treatment was verified by comparing the forcedisplacement curves of two tensile tests before and after laser engraving (Figure 9). These two curves are similar: The elastic stiffness measured at room temperature is 12.1 and $12.2 \mathrm{kN} / \mathrm{mm}$, respectively. With a relative difference of $0.1 \mathrm{kN} / \mathrm{mm}$, it is clear that the laser source did not influence the mechanical behaviour of the TA6V material.

(a)

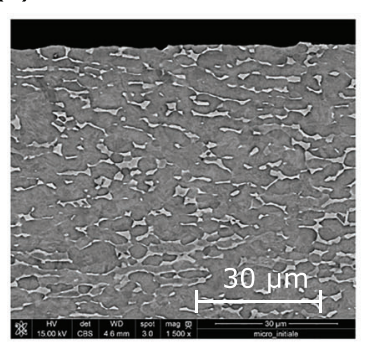

(b)

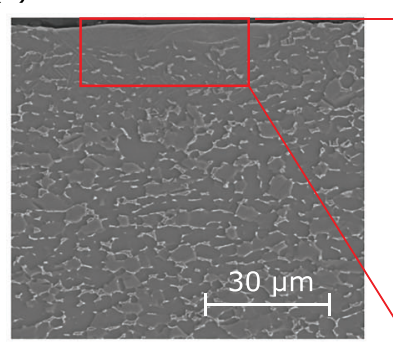

(c)

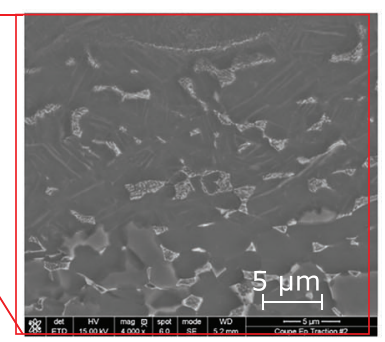

F I G U RE 8 Cross-sectional view of TA6V sample microstructure. (a) Initial. (b) On a speckle made by the laser engraving technique (M5). The speckle was $80.62 \mu \mathrm{m}$ in diameter. (c) Microstructure observed on a speckle (in red)

F I G U RE 9 Mechanical behaviour of two TA6V samples: Sample without speckle and sample speckled by laser engraving (M4). Tensile tests were performed at room temperature

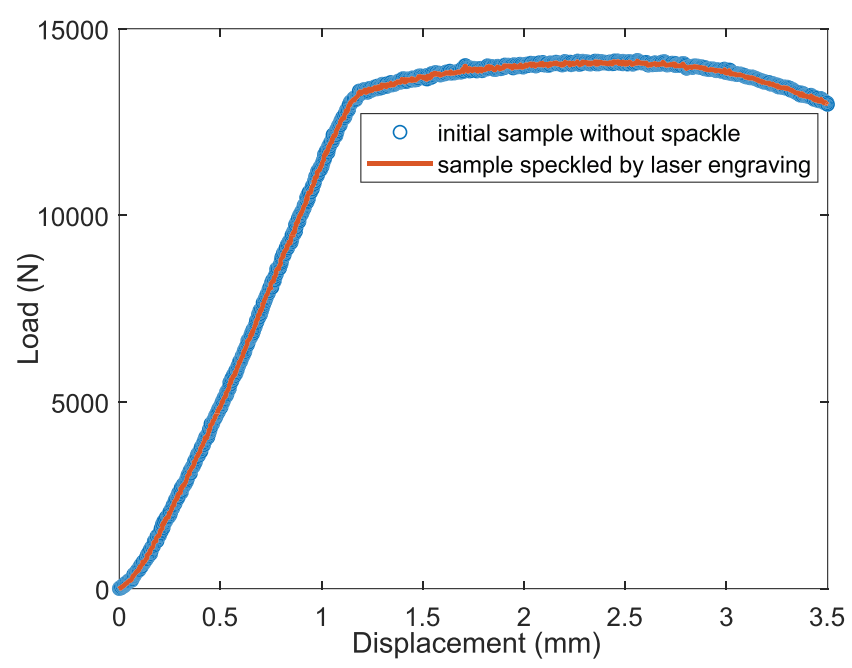


Following characterisation of TA6V material speckled by a laser source, Figure 10a-c (the first row) shows images of speckles made by the techniques M4, M5 and M6 at $25^{\circ} \mathrm{C}$, respectively. Their morphological results are presented in Table 4.

The speckle radii measured from techniques M4, M5 and M6 are closer to the numerical speckle radius than those made using M1, M2 and M3: They are $114 \pm 200,139 \pm 50$ and $89 \pm 115 \mu \mathrm{m}$, respectively. Speckle distances obtained from these three techniques are similar $(185 \pm 70 \mu \mathrm{m})$, but higher than the numerical equivalent $(30 \mu \mathrm{m})$. The speckle densities resulting from techniques M4, M5 and M6 are 0.22, 0.39 and 0.29, respectively, lower than the numerical speckle density of 0.4 .

Speckles created using the laser source (M4, M5 and M6) are relatively similar to the target numerical speckle. Moreover, although the TA6V sample surfaces are slightly changed by the laser-matter interaction (Figure 8), the mechanical behaviour of the TA6V material after being patterned using a laser setting of $P=20 \mathrm{~W}, S=80 \mathrm{~mm} / \mathrm{s}$ remains unchanged (Figure 9).

\subsection{Image quality at different temperatures}

The speckle image quality is evaluated on the basis of three criteria: MIG, Shannon entropy and the percentage of saturated pixels. The image quality of the six speckle patterns was then compared at different temperatures: room

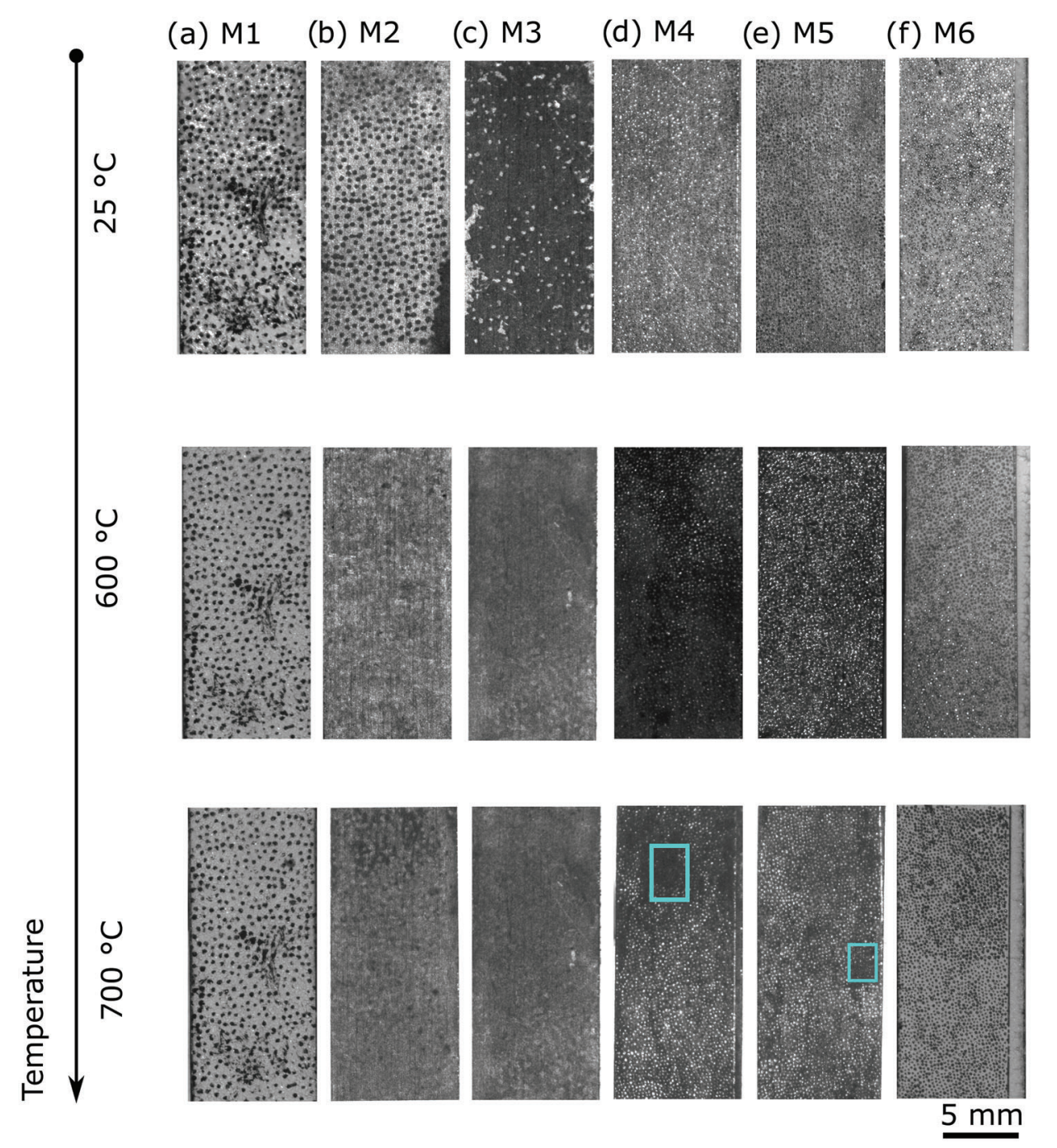

F I G U R E 10 Speckle images made by six techniques: (a) M4 (laser engraving), (b) M5 (combined anodisation and laser), (c) M6 (combined anodisation and painting), (d) M4 (laser engraving), (e) M5 (combined anodization and laser engraving) and (f) M6 (combined painting and laser engraving) at three temperatures: 25,600 and $700^{\circ} \mathrm{C}$ 
temperature (25), 600 and $700^{\circ} \mathrm{C}$. Figure 10 presents the images of six types of speckles patterns at three temperatures: 25,600 and $700^{\circ} \mathrm{C}$. The MIG, entropy and percentage of pixel saturation of six types of speckle patterns are shown in Table 5, followed by the relative reduction of the MIG, entropy and pixel saturation of these six patterns at 600 and $700^{\circ} \mathrm{c}$ compared with those at $25^{\circ} \mathrm{C}$ (Table 6).

\subsubsection{Image quality at room temperature $\left(25^{\circ} \mathrm{C}\right)$}

Images of speckles made using the 'additive' and 'subtractive' methods at room temperature are presented in Figure 10a-c and Figure 10d-f, respectively (the first row of Figure 10). Speckles made using the 'subtractive' methods present specular reflection, inducing saturated pixels on images. The glare was quantified by the percentage of saturated pixels (Table 5). Speckles made using techniques M1, M2, M3 and M5 have less than $1 \%$ of saturated pixels, while speckles made using M4 and M6 have 2\% and 4\% of saturated pixels, respectively. This glare is probably from light reflecting off laserproduced speckle dots. The glare could lead to disturbances when correlating. Among the six techniques, speckles made using M2 and M5 presented the highest MIG values. Speckles made using M1 and M2 have the highest entropy values (7.4). Speckles made using M5 have a slightly lower entropy value (6.9). Considering all image quality parameters, speckles made using technique M5 (combined laser engraving and anodisation) and M2 (inverse anodisation) present the best quality at room temperature: high MIG and entropy values and a low percentage of saturated pixels.

\subsubsection{Speckle quality at $600^{\circ} \mathrm{C}$}

The second row in Figure 10 shows six types of speckle patterns at $600^{\circ} \mathrm{C}$. With the same exposure time as that used at room temperature, the speckle patterns are less visible. Image contrast, characterised by MIG and entropy parameters, is

TA B L E 5 Comparison of MIG, entropy and the percentage of saturated pixels for six speckle production methods (M1-M6) at (i) 25, (ii) 600 and (iii) $700^{\circ} \mathrm{C}$

\begin{tabular}{|lrrrrrllllll|}
\hline \multicolumn{1}{l}{ Parameters } & \multicolumn{1}{c}{ MIG } & \multicolumn{3}{c}{ Entropy } & \multicolumn{3}{c}{ \% saturated pixels } \\
\hline Temperature $\left({ }^{\circ} \mathrm{C}\right)$ & \multicolumn{1}{c}{25} & 600 & 700 & 25 & 600 & 700 & 25 & 600 & 700 \\
\hline Methods & M1 & 9.8 & 7.6 & 7.6 & 7.4 & 6.7 & 6.7 & 1.0 & 0 & 0 \\
& M2 & 15.8 & 12.0 & 7.4 & 7.4 & 6.6 & 6.1 & 0.8 & 0.1 & 0.1 \\
& M3 & 7.7 & 5.9 & 5.9 & 6.1 & 6.1 & 5.9 & 0.15 & 0 & 0 \\
M4 & 13.8 & 5.2 & 8.7 & 6.7 & 5.9 & 6.4 & 1.83 & 0.04 & 0 \\
M5 & 15.9 & 12.4 & 10.7 & 6.9 & 6.6 & 6.5 & 0.4 & 1 & 0.1 \\
M6 & 14.3 & 11.3 & 9.7 & 6.8 & 6.7 & 6.6 & 4 & 0 & 0 \\
\hline
\end{tabular}

Note: The six methods are M1 (painting), M2 (direct anodisation), M3 (inverse anodisation), M4 (laser engraving), M5 (combined anodisation and laser engraving) and M6 (combined painting and laser engraving).

TA B LE 6 MIG and entropy relative reductions (in \%) of six speckle patterns (M1-M6) at (i) 600 and (ii) $700^{\circ} \mathrm{C}$ compared with those at room temperature

\begin{tabular}{|c|c|c|c|c|c|}
\hline \multicolumn{2}{|c|}{$\begin{array}{l}\text { Parameters } \\
\text { Temperature }\left({ }^{\circ} \mathrm{C}\right)\end{array}$} & \multicolumn{2}{|c|}{ Relative reduction in MIG (\%) } & \multicolumn{2}{|c|}{ Relative reduction in entropy (\%) } \\
\hline \multirow[t]{4}{*}{ Methods } & M1 & 24 & 24 & 10 & 10 \\
\hline & M2 & 24 & 53 & 11 & 18 \\
\hline & M3 & 23 & 23 & 3 & 3 \\
\hline & M6 & 21 & 32 & 3 & 2 \\
\hline
\end{tabular}

Note: The six speckle production methods are M1 (painting), M2 (direct anodisation), M3 (inverse anodisation), M4 (laser engraving), M5 (combined anodisation and laser engraving) and M6 (combined painting and laser engraving). 
lower, which is likely to be due to TA6V surface oxidation. For example, saturated pixels observed on the M4 and M6 samples disappeared, while the saturation of pixels increased by $1 \%$ for the M5 sample because the thickness of the oxidation layer in each sample is different. It should be recalled that when using technique M5, the TA6V surface is pre-oxidised to reduce the problem of glare. The speckle pattern M4 was the most affected by oxidation: Its MIG value is 5.2 (Table 5), 62\% lower than that at room temperature (Table 6). Furthermore, due to oxidation, the speckle patterns made using M2 cannot easily be seen by eyes. Its MIG value is $24 \%$ lower than at room temperature. The MIG values of speckles made using the other methods (M1, M5 and M6) are 21\%-22\% lower. The same tendency is observed in the relative decreases in entropy values. Among the five methods, the speckle pattern made using M5 presents the best contrast (highest values of MIG and entropy and the least reduction in MIG and entropy). This technique produces the best speckle quality at $600^{\circ} \mathrm{C}$.

\subsubsection{Speckle quality at $700^{\circ} \mathrm{C}$}

At $700^{\circ} \mathrm{C}$, speckles made using techniques M2 and M3 (the third row in Figure 10) are barely visible to the naked eye. Furthermore, speckle patterns made by M4 and M5 techniques are hardly seen by eyes on some regions (highlighted with green rectangles in Figure 10), which may cause correlation disruptions. The results in Table 5 show that their MIG and entropy values continued to decrease. Among the six sets of speckles, those made using M2 and M4 are the most influenced by oxidation. The MIG values decrease by $53 \%$ with M2 and by $37 \%$ with M4. The MIG value of speckles made using method M5 decreased by 33\%. The entropy of speckles produced by M1 and M2 was significantly reduced. Nevertheless, speckles made using techniques M1 and M6 are still visible and present the best contrast (highest entropy values). The MIG values decreased by $22 \%$ and $32 \%$, respectively (Table 6). This can be explained by the Vitcas white paint covering the TA6V samples. The paint protected the TA6V surface from oxidation.

\subsection{Speckle adherence at high temperatures}

The speckles made using techniques M1, M4, M5 and M6, presenting the best image quality at 600 and $700^{\circ} \mathrm{C}$, were chosen to evaluate the adherence of speckles to the TA6V material. Figure 11 presents the strain fields from TA6V

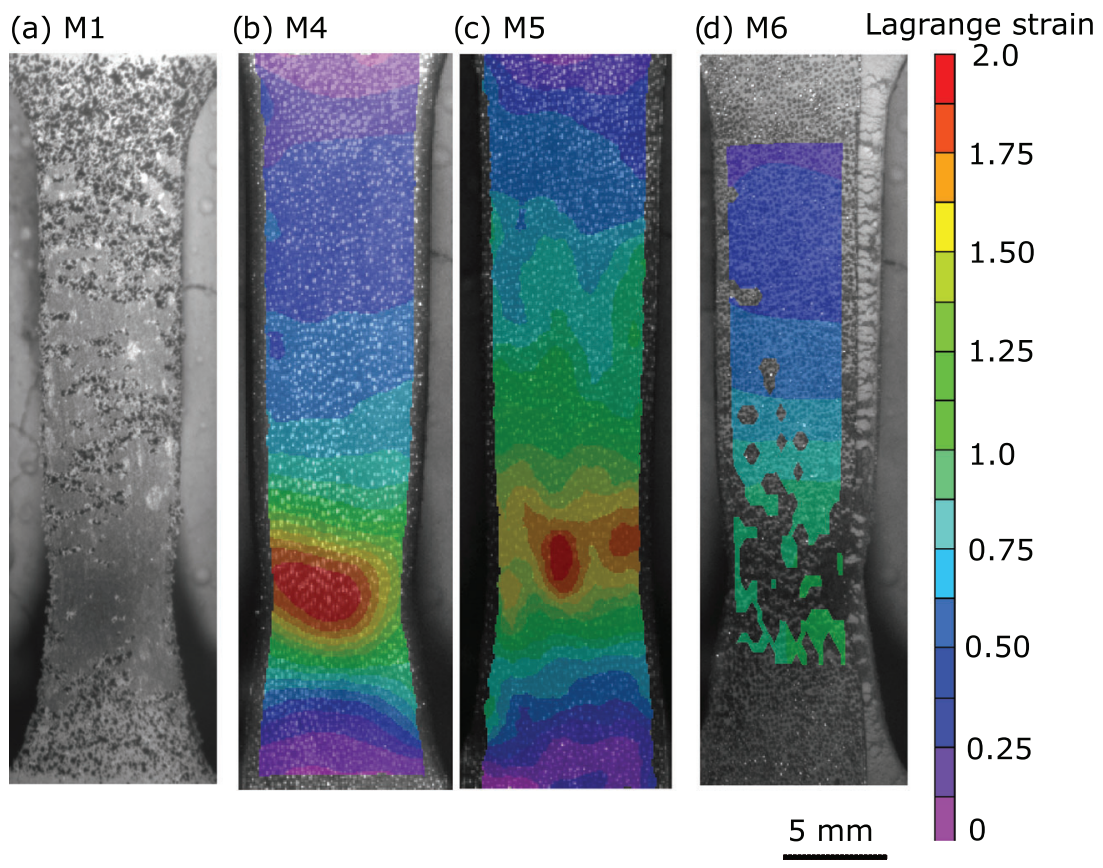

F I G U R E 11 Longitudinal strain fields from tensile tests performed on TA6V samples speckled using different techniques: (a) M1 (painting), (b) M4 (laser engraving), (c) M5 (combined anodisation and laser engraving) and (d) M6 (combined painting and laser engraving) at $600^{\circ} \mathrm{C}$. With the same amount of strain as M4 and M5, the speckles M1 and M6 come off, completely causing decorrelation problem 
tensile tests at $600^{\circ} \mathrm{C}$ on samples speckled using the four techniques listed above. With the same amount of longitudinal strain applied on the specimen, speckle patterns made by techniques M1 and M6 flake off completely that make the DIC correlation disrupted (Figure 11a,d). These two speckle patterns resist only a strain of about 22\%. Meanwhile, the adherence of the speckle patterns made by techniques M4 and M5 on the TA6V material is outstanding. They can resist for a maximum strain of $200 \%$ (Figure $11 b, c)$.

\section{4 | DISCUSSION}

\section{1 | Possibility of making a repeatable speckle pattern}

Previous authors have documented the importance of speckles in improving spatial resolution and reducing DIC errors. $^{[3-4,12]}$ They recommend that certain characteristics of speckle morphology such as speckle size, distance and density should be respected. However, in practice, such requirements are quite difficult to achieve because, in most current speckle preparation techniques, speckle morphology is neither really controlled nor repeatable. In our study, we suggest novel techniques that produce repeatable speckles meeting speckle criteria in order to increase the resolution of strain measurements. Controlled speckles are made from the numerical model using six different techniques. They are classified into two groups. The 'additive' techniques use a polymer mask and a lithographical technique (M1, M2 and M3), while the 'subtractive' techniques use a laser source (M4, M5 and M6).

In the first group, polymer mask production is critical. Section 3.1.1 shows that nine experiments to find a set of laser parameters for producing the mask were insufficient. The vinyl polymer chosen for the mask is an unconventional material for use with a laser. Analysis of variance in the factors influencing the radius of the holes made in the polymer showed that three parameters-laser power, laser scanning speed and laser scanning strategy-and their interactions influence hole size, and thus, speckle radius produced using techniques M1, M2 and M3. These results are indeed consistent with studies on the interaction between lasers and matter, which is well known in the SLM process. ${ }^{[24]}$ The energy emitted by a laser is proportional to its power and scanning speed. The results of the above experiment finally enabled us to successfully produce the polymer mask. Polymer masks produced using laser parameters $P=30 \mathrm{~W}$, $V=80 \mathrm{~mm} / \mathrm{s}$ do not present local damage to the polymer and are thus sufficiently hard to be manipulated. To the authors' knowledge, this is the first publication in speckle techniques involving a polymer mask with a hole size of less than $1 \mathrm{~mm}$. However, the hole radius, density and distance on the polymer mask differ from those on the numerical equivalent. It is necessary to maintain a minimum distance of at least $320 \mu \mathrm{m}$ between speckle dots when producing a polymer mask; the spatial resolution of kinematic fields measured by DIC is thereby reduced. DIC spatial resolution could be improved by performing additional experiments in order to determine optimised laser parameters.

Three speckle patterns, M1, M2 and M3, were created with the polymer mask. Consequently, these speckles have larger distances between dots and lower densities than the numerically produced pattern. Globally, the polymer mask does create speckle patterns on TA6V sample surfaces using techniques M1 and M2, except that some marks remain on the TA6V samples. Their speckle morphology (radii) is slightly better than speckles made using air spraying techniques. ${ }^{[12]}$ Moreover, speckle sizes are controllable. The contribution of this study is to demonstrate the possibility of creating speckles on the basis of lithographical techniques and of achieving a better resolution. We understand that the numerical method of producing repeatable speckles has been studied by Mazzoleni et al. ${ }^{[19]}$ and Chen et al. ${ }^{[20]}$ These authors used laser printing and toner transfer processes to create speckles with diameters varying between 0.2 and $1.2 \mathrm{~mm}$. More interestingly, previous studies show a difference between a numerical speckle and a real one, which is in line with our results. Small speckles are difficult to prepare due to the toner transfer process.

Nevertheless, our results relating to speckle quality made using three techniques involving a polymer mask show some limits. For instance, speckles made by technique M3 have a low speckle density which reduces spatial resolution. This could be explained by the high viscosity of Jelt varnish, which is made of kerosene disulphide from processed oil, whose viscosity is greater than that of water. Improving the speckle production techniques used in our study would require further investigation into (i) controlling Jelt varnish droplet size and (ii) reducing varnish viscosity.

The last three types of speckle (M4, M5, M6) were made using a laser source. These speckles are more precise and closer to the numerical speckle pattern thanks to the advantages of the laser system. Compared with the other three techniques, speckle production processes involving laser engraving require fewer steps and are thus less costly and time consuming. Many studies use laser engraving techniques because of their high precision and rapidity. For example, Hu et al. ${ }^{[15]}$ use this technique to produce a speckle size of $5 \mu \mathrm{m}$ in order to increase DIC spatial resolution. Although laser 
engraving is not a new technique for creating speckles, our study contributes to confirming the integrity of the material's microstructure and mechanical behaviour after the laser engraving process. These parameters should be verified systematically after laser engraving. Surface observations made after laser engraving suggest a possible reason for the glare problem that occasionally occurs on metal materials. Moreover, our studies suggest that combinations of techniques, for example, laser engraving and anodisation, create speckles with better contrast than those involving a single technique.

\section{2 | Speckle quality at various temperatures and guidelines to choose appropriate speckle production techniques for TA6V tensile test specimens at different temperatures}

Previous studies have shown that speckle quality may be assessed using many parameters. These can be grouped into two classes: (i) morphological quality, including speckle radius, distance (speckle spacing) and density, and (ii) image quality, including MIG value and the greyscale dynamic range represented by the entropy value and the percentage of saturated pixels.

Morphological quality is usually used to assess DIC spatial resolution and error (both random and systematic). Several studies have demonstrated the relationship between speckle size and the subset size used in the local DIC. For example, Lecompte et al. ${ }^{[25]}$ investigated the influence of speckle radii, speckle densities and subset sizes on the accuracy of the displacement field obtained by local DIC. Results showed that a speckle density varying between 0.4 and 0.7, a speckle radius of five pixels and a subset of 15 pixels give the most accurate DIC displacement. Furthermore, Crammond et al. ${ }^{[26]}$ explained that global parameters such as Shannon entropy are insufficient to assess speckle quality. These authors highlight the fact that morphological quality, including speckle density and speckle size, should be considered as additional parameters in evaluating DIC precision. Moreover, numerical modelling studies by Bornert et al. ${ }^{[27]}$ on the effect of speckle size on displacement error in the ultimate error regime (when DIC error is no longer dependent on subset shape function) also demonstrated that for a given subset size, a lower speckle radius could decrease displacement error thanks to more information being contained in a subset. Finally, Reu et al. ${ }^{[3-6]}$ suggested using speckle distance as an additional morphological parameter in the analysis of speckle quality. These authors recommend a speckle distance of two pixels to avoid the problem of aliasing and also published guidelines for a 'good' or 'ideal' speckle for use with DIC.

As a complement to morphological parameters, speckle quality is assessed by image quality criteria: MIG and entropy. Dong et al. ${ }^{[12]}$ consider MIG to be a 'global' parameter, characterising global image contrast. ${ }^{[22]}$ However, MIG does not consider the influence of local fluctuation and image noise. An image with a lot of noise or subject to aliasing can have a high MIG value and yet not show good contrast. To overcome the drawback of using the MIG criterion, the sum of square of subset intensity gradient (SSSIG) could be used instead. The second image quality parameter, entropy, represents the breadth of grey scale dynamic range. A high-contrast image should have a wide dynamic range and thus a high entropy value. However, entropy does not characterise the local features of speckles. It should be noted that the three criteria chosen for speckle quality in this study (the MIG, Shannon entropy and percentage of pixel saturation) depend on experimental condition, for example, the lighting and the exposure time of the camera. Therefore, they would change if condition changes. Nevertheless, these three criteria are used to compare relatively the speckle quality made by six different techniques to find an appropriate speckle patterns meeting requirements in this study.

To summarise, a 'good' speckle should satisfy the following conditions: a speckle radius of between three and five pixels, a speckle density of between 0.4 and 0.5 , a speckle distance of at least two pixels, the highest possible MIG and entropy values and the lowest possible number of saturated pixels. The limit of recent research into speckles is that each criterion, with its advantages and drawbacks, means that it is not possible to have a general method that combines all speckle criteria. In this study, speckle quality is assessed objectively and globally by considering all aspects of speckles. With given criteria for a 'good' speckle, speckle quality resulting from six techniques is compared to find the appropriate speckle production method at different temperatures.

At room temperature, speckle densities produced using polymer mask techniques M1 and M2 are slightly lower than those produced by the laser engraving techniques M4, M5 and M6. Speckle distances produced using M1, M2 and M3 are also greater than those using techniques M4, M5 and M6. In terms of speckle quality, techniques M2 (direct anodisation) and M5 (combined anodisation and laser engraving) produce the best results: Speckle morphology is close to recommended levels, and the highest contrast values are achieved. Their MIG and entropy values are of the same order of magnitude as speckles made by classical painting techniques. ${ }^{[12]}$ The technique combining anodisation and laser engraving (M5) suggests a novel method resulting in high speckle quality. 
Nevertheless, at room temperature, a high level of specular light is observed on speckles made using techniques M4 and M6 due to the crater-like shape of the speckle dots. The glare can cause a serious decorrelation in DIC. Some studies suggest solutions to deal with this. For example, Lepage et al. ${ }^{[28]}$ suggest using a cross-polarisation method to attenuate the glare. Results show that setting cross polarisation on the camera and using LED lighting reduced the 2D-DIC displacement error by $52 \%$. However, in their study, the experimental set-up required two LEDs at different angles. This condition could not be satisfied in our tensile test experiment since the observation window was only $20 \mathrm{~mm}$ wide and $150 \mathrm{~mm}$ high. Poncelet et al. ${ }^{[29]}$ also demonstrate that DIC error is produced near the saturation zone because of light reflection. They suggest using the multiple illumination DIC algorithm to correct the glare problem. In this algorithm, the missing information in the saturated region is compensated for by other images from an additional illumination source and by weighting functions. However, this method uses multiple lights which might not be appropriate to our experimental set-up. That is why in this study a large subset window of $57 \times 57$ pixels was chosen to deal with the glare problem. Since the region of saturated pixels was from five to seven pixels, the large subset could cover saturated regions, thus avoiding the problem of decorrelation in local DIC. As a result, the spatial resolution of the kinematic fields would be reduced. Further methods for reducing glare should be investigated in order to improve spatial resolution and reduce DIC error.

At $600^{\circ} \mathrm{C}$, image quality is reduced. It is supposed that this is due to the oxidation of white paint and to titanium oxidation. Consequently, the MIG value of speckles made using technique M3 (inverse anodisation) was significantly reduced (by 62\%). For the other laser engraving techniques, the reduction exceeded $20 \%$. The MIG and entropy values are lower than results obtained in many previous studies on speckles made by painting at high temperature. ${ }^{[12]}$ On the basis of the various criteria, the speckles produced using technique M5 present the best quality. Furthermore, these speckles show excellent adherence. The speckle pattern resists a strain of $200 \%$, which is much better than that obtained by painting techniques. ${ }^{[9-11]}$ The adherence of this speckle pattern is as good as that obtained by the electrochemical marking technique. ${ }^{[13,14]}$ The experimenter should use this technique for TA6V tensile tests between 20 and $600^{\circ} \mathrm{C}$ to access further information about the behaviour of the material by measuring strain fields.

At $700^{\circ} \mathrm{C}$, image contrast decreases considerably, probably from the acceleration of TA6V sample oxidation. It is particularly noteworthy on samples without painting (M2-M5) that could induce DIC errors or even decorrelation problems. At $700^{\circ} \mathrm{C}$, only the speckle patterns made by painting techniques, M1 and M6, are visible and present the best contrast. The experimenter should choose between these two techniques to create speckles on their sample. However, speckles flake off at strains of 0.2. Painting techniques can resist high temperature and provide a stable, high-contrast image quality but cannot withstand high levels of strain.

Throughout this study, it may be remarked that no speckle preparation technique can provide the recommended speckle morphology, high contrast, resistance to high temperature and high strain (up to a strain of 2.0) for all temperatures at the same time. A mechanical experimenter should use a speckle technique that compromises between the requirements of spatial resolution, image contrast and maximum strain value. From 20 to $600^{\circ} \mathrm{C}$, the speckle pattern produced by M5 technique can meet speckle criteria (speckle morphology and 'good' contrast) and excellent adherence on the specimen surface: Strains of more than $200 \%$ can be measured. From $700^{\circ} \mathrm{C}$ and above, the speckle patterns obtained by M1 and M6 techniques show a better contrast than others technique, but it allows the measurement of strain only up to $22 \%$. Our studies provide guidelines for choosing appropriate speckling production techniques depending on experimental temperature and strain limit measurements. However, this study does have some limitations that should be addressed. For example, only three criteria, MIG, entropy and the percentage of saturated pixels, are used to assess image quality. Speckle quality would be evaluated more objectively using a global model that includes every speckle characteristic. Furthermore, the hypothesis that oxidation reduces image quality on all speckle patterns requires confirmation. Further investigation is required into how the thickness of the oxide layer affects image contrast. According to Kumar et al., ${ }^{[30]}$ it is possible to predict the thickness of oxide layers created on TA6V. Image contrast can be indirectly inferred from oxide layer thickness. This kind of correlation model could help an experimenter to estimate speckle quality as a function of temperature and time.

\section{5 | CONCLUSION}

This study introduces six types of speckle preparation techniques to measure strain during tensile tests of TA6V specimens at high temperatures. Repeatable speckles are produced using a numerical speckle generator to allow objective comparison of speckle quality at different temperatures in relation to several criteria. 
The techniques are classified into two groups: 'additive' (M1, M2 and M3), using a polymer mask and lithographybased method, and 'subtractive' (M4, M5 and M6), using a laser source.

The first step in the 'additive' methods is to create a useable polymer mask using a laser source. To that end, nine experiments were performed, combining three laser parameters (power, scanning speed and strategy) at various levels. The final set of parameters is: strategy: contour and hatch, laser power $30 \mathrm{~W}$ and scanning speed $80 \mathrm{~mm} / \mathrm{s}$. In this study, the 'subtractive' methods create speckle patterns using a laser source set to power $20 \mathrm{~W}$ and scanning speed $80 \mathrm{~mm} / \mathrm{s}$. Speckle quality is then analysed using six criteria: three morphological and three relating to image quality. For speckles produced by techniques from the first group, a difference in speckle morphology was observed between the real and the numerical samples. The techniques in the second group produce real speckle dots that are quite similar to their numerical counterparts thanks to the high precision of the laser system.

At room temperature, regardless of the adherence of speckle patterns on the TA6V material, the speckle patterns made using techniques M2 (direct anodisation) and M5 (combined anodisation and laser) present the 'best' quality in terms of morphology and image quality criteria. At $600^{\circ} \mathrm{C}$, the speckles made by technique M5 gave the best contrast (MIG: 12.35, entropy: 6.86 and good adherence on the TA6V surface: the measured longitudinal strain was 200\%) although saturated pixels appeared on the TAV6 surface due to surface oxidation. At $700^{\circ} \mathrm{C}$, the speckles produced using M1 (painting) and M6 (combined laser engraving and painting) techniques show a higher contrast than the other techniques but only allow the longitudinal strain measurement of $22 \%$.

Perspectives for further studies of speckle production techniques at high temperature are:

- to improve experimental design in order to optimise laser parameters for a better polymer mask,

- to analyse paint droplet size in order to improve speckle densities,

- to study the nature of the oxidation layers on TA6V at high temperature that reduce speckle image quality and to correlate image contrast with oxide layer thickness at different temperatures.

- to develop other speckle methods that meet 'good speckle' criteria and good surface adherence, for example, the golden coating method coupled with laser engraving.

\section{ACKNOWLEDGEMENTS}

The authors would like to begin by thanking the IMT Mines Albi engineering school and the Occitanie Region for financial aid towards this study ('program 09A61 909 9092, Dossier: 2017 001838'). We would also like to offer special thanks to all the researchers and technicians in the ICA laboratories for their technical help and scientific advice.

\section{NOMENCLATURE}

C contour: the laser path is circular and the hatch spacing is $80 \mu \mathrm{m}$

d distance between speckle dots $(\mu \mathrm{m})$

DIC digital image correlation

$\mathrm{dR}$ difference between the actual radius of speckle dots made using different techniques and the target radius $(\mu \mathrm{m})$

$\mathrm{H} \quad$ combination of hatching and contouring

$+\mathrm{C}$

$\mathrm{H} \quad$ hatch: the laser path scanning is parallel and the hatch spacing is $80 \mu \mathrm{m}$

M1 technique producing speckles by painting

M2 technique producing speckles by direct anodisation

M3 technique producing speckles by inverse anodisation

M4 technique producing speckles by laser engraving

M5 technique producing speckles by combining laser engraving and anodisation

M6 technique producing speckles by combining painting and laser engraving

MIG mean intensity gradient

$\mathrm{P} \quad$ laser power (W)

$\mathrm{r} \quad$ speckle radius measured by the digital image processing procedure

$\mathrm{S} \quad$ laser strategy: different laser path scanning strategies

$\mathrm{V} \quad$ laser scanning speed $(\mathrm{mm} / \mathrm{s})$

ZOI zone of interest 


\section{ORCID}

Phuong Luong (1) https://orcid.org/0000-0002-3967-0744

Rébecca Bonnaire (iD https://orcid.org/0000-0003-2085-2254

Jean-Noel Périé (i) https://orcid.org/0000-0002-1522-3931

Quentin Sirvin (D) https://orcid.org/0000-0003-0547-5278

Luc Penazzi (1) https://orcid.org/0000-0002-3989-0069

\section{REFERENCES}

[1] M. Rollin, L. Penazzi, V. Velay, A. Dupuy, S. Gallet, Int. J. Mech. Sci. 2018, 141, 479.

[2] M. A. Sutton, J.-J. Orteu, H. Schreier, Image Correlation for shape, motion and deformation measurements: Basic concepts, theory and applications, Springer, Boston 2009.

[3] P. Reu, Exp. Tech. 2014a, 38, 1.

[4] P. Reu, Exp. Tech. 2014b, 38, 1.

[5] P. Reu, Exp. Tech. 2015, 39, 1.

[6] P. Reu, Exp. Tech. 2015, 39(1), 1.

[7] J. L. Turner, S. S. Russell, Strain 1990, 26, 55.

[8] J. S. Lyons, J. Liu, M. A. Sutton, Exp. Mech. 1996, 36, 64.

[9] B. Pan, D. Wu, Y. Xia, Opt. Lasers Eng. 2010, 48, 841.

[10] J. T. Hammer, J. D. Seidt, A. Gilat, Proceedings of the Society for Experimental Mechanics Series 3, Springer, New York $2014,167$.

[11] B. Chartrel, Ph.D. Thesis, Université Paris Sciences et Lettres 2016.

[12] Y. L. Dong, B. Pan, Exp. Mech. 2017, 57, 1161.

[13] M. Vautrot, P. Balland, O. S. Hopperstad, L. Tabourot, J. Raujol-Veillé, F. Toussaint, Exp. Mech. 2014, $54,1163$.

[14] Q. Sirvin, V. Velay, R. Bonnaire, L. Penazzi, J. Manuf. Process. 2019, $38,472$.

[15] Y. J. Hu, Y. J. Wang, J. B. Chen, J. M. Zhu, Exp. Tech. 2018, 42, 523.

[16] Q. Zheng, N. Mashiwa, T. Furushima, Mater. Des. 2020, 191, 108626.

[17] M. Vanderhasten, Ph.D. Thesis, KU Leuven 2007.

[18] J.-J. Orteu, D. Garcia, L. Robert, F. Bugarin, in Proceedings of the International Society of Optical Engineering (SPIE), 2006, 634110H.

[19] P. Mazzoleni, E. Zappa, F. Matta, M. A. Sutton, Opt. Lasers Eng. 2015, 75, 72.

[20] Z. Chen, C. Quan, F. Zhu, X. He, Meas. Sci. Technol. 2015, 25, 095201.

[21] J. Goupy, L. Creighton, Introduction aux plans d'expériences, Dunod, Paris 2006.

[22] B. Pan, Z. Lu, H. Xie, Opt. Lasers Eng. 2010, 48, 469.

[23] International Digital Image Correlation Society, in A Good Practices Guide for Digital Image Correlation, (Eds: E. M. C. Jones, M. A. Ladicola), iDICs: International Digital Image Correlation society, 2018. https://idics.org/

[24] Z. Yan, W. Liu, Z. Tang, X. Liu, N. Zhang, M. Li, H. Zhang, Opt. Laser Technol. 2018, 106, 427.

[25] D. Lecompte, H. Sol, J. Vantomme, H. Habraken, Opt. Lasers Eng. 2006, 44, 1132.

[26] G. Crammond, S. W. Boyd, J. M. Dulieu-Barton, Opt. Lasers Eng. 2013, 54, 1368.

[27] M. Bornert, F. Brémand, P. Dumalin, J. C. Dupré, M. Fazzini, M. Grédiac, F. Hild, S. Mistou, J. Molimard, J.-J. Orteu, L. Robert, Y. Surrel, P. Vacher, B. Wattrisse, Exp. Mech. 2009, 49, 353.

[28] W. S. Lepage, S. H. Daly, J. A. Shaw, Exp. Mech. 2016, 56, 969.

[29] M. Poncelet, H. Leclerc, Exp. Mech. 2015, 55, 1317.

[30] S. Kumar, T. S. N. Narayanan, S. G. Raman, S. K. Seshadri, Mater. Chem. Phys. 2010, 119, 337. 